\title{
Desarrollo de una bebida energizante con pulpa de maracuyá, cristales de aloe vera, panela y propiedades antioxidantes
}

\section{Development of an energy drink with passion fruit pulp, aloe vera crystals, panela and antioxidant properties}

\author{
Jhon Edinson Nieto-Calvache ${ }^{1 *}$; Claudia Andrea Suarez-Ramos ${ }^{2}$
}

1'Ing. Agroindustrial, Esp., M.Sc., Ph.D. Universidad del Cauca, Facultad de Ciencias Agrarias. Popayán - Cauca, Colombia; e-mail: jnieto@unicauca.edu.co; (Dhttps://orcid.org/0000-0003-1966-8636

${ }^{2}$ Ing. Agroindustrial, M.Sc. Servicio Nacional de Aprendizaje SENA, Centro Agropecuario, regional Cauca. Popayán - Cauca, Colombia; e-mail: casuarezr@ sena.edu.co; Dhttps://orcid.org/0000-0002-7143-8697

*autor para correspondencia: jnieto@unicauca.edu.co

Cómo citar: Nieto-Calvache, J.E.; Suarez-Ramos, C.A. 2020. Desarrollo de una bebida energizante con pulpa de maracuyá, cristales de aloe vera, panela y propiedades antioxidantes. Rev. U.D.C.A Act. \& Div. Cient. 23(2):e1456. http://doi.org/10.31910/rudca.v23. n2.2020.1456

Artículo de acceso abierto publicado por Revista U.D.C.A Actualidad \& Divulgación Científica, bajo una licencia Creative Commons CC BY-NC 4.0

Publicación oficial de la Universidad de Ciencias Aplicadas y Ambientales U.D.C.A, Institución de Educación Superior Acreditada de Alta Calidad por el Ministerio de Educación Nacional.

Recibido: Enero 18 de 2020 Aceptado: Septiembre 7 de 2020 Editado por: Ingeborg Zenner de Polanía

\section{RESUMEN}

En los últimos años, la demanda de productos saludables se ha venido incrementando, con lo cual, muchas investigaciones se han focalizado hacia la producción de alimentos y bebidas con potencial nutracéutico. El objetivo de este trabajo fue desarrollar una bebida energizante, a base de panela, jugo de maracuyá, cristales de aloe vera (AV), con propiedades antioxidantes. Se evaluó el contenido de compuestos fenólicos, ácido ascórbico y la capacidad antirradicalaria de las materias primas y las bebidas producidas. Se realizó un análisis sensorial, para verificar la aceptación del sabor, color, textura del AV y la aceptabilidad global de tres bebidas, seleccionadas de acuerdo con sus propiedades antioxidantes. Los resultados mostraron que la panela tenía el mayor contenido de compuestos fenólicos (59,4 \pm 0,2mg AGE/g), mientras que el jugo de maracuyá, la mayor actividad antirradicalaria $(657 \pm 5 \mu \mathrm{g}$ eq $\mathrm{AA} / \mathrm{mL})$. Las bebidas analizadas dentro del diseño experimental variaron su actividad antioxidante, con la variación de los factores. De las tres bebidas seleccionadas, la bebida 2 presentó la mayor capacidad antirradicalaria (419 \pm $1 \mu \mathrm{g}$ eq $\mathrm{AA} / \mathrm{mL})$ y contenido de vitamina $\mathrm{C}(15,75 \pm 0,03 \mu \mathrm{g} / \mathrm{mL})$ y, además, un importante contenido de compuestos fenólicos (7,6 $\pm 2 \mathrm{mg}$ AGE/mL). Asimismo, los resultados del panel sensorial mostraron que la bebida 2 tenía una alta aceptabilidad global y una mayor aceptación del sabor, por lo cual, se puede concluir que esta bebida, es la que presenta mayor potencial antioxidante y comercial.

Palabras clave: Aloe vera (L.) Burm. F.; Aloína; Compuestos fenólicos; Capacidad antirradicalaria; Panel sensorial.

\section{ABSTRACT}

In recent years, the demand for healthy products has been increasing, so much research has focused on the production of foods and beverages with nutraceutical potential. The aim of this work was to develop an energy drink, based on panela, passion fruit juice and aloe vera (AV) crystals with antioxidant properties. The content of 
Nieto-Calvache, J.E.; Suarez-Ramos, C.A.: Bebida con potencial antioxidante

phenolic compounds, ascorbic acid and the anti-radical capacity of the raw materials and beverages produced were evaluated. A sensory analysis was performed, to verify the acceptance of taste, color, AV texture and overall acceptability of three beverages, selected according to their antioxidant properties. The results showed that panela had the highest content of phenolic compounds (59.4 \pm $0.2 \mathrm{mg} \mathrm{AGE} / \mathrm{g}$ ), while passion fruit juice had the highest anti-radical activity $(657 \pm 5 \mu \mathrm{g}$ eq $\mathrm{AA} / \mathrm{mL})$. The beverages analyzed within the experimental design varied in their antioxidant activity with varying factors. Of the three drinks selected, drink 2 had the highest antiradical capacity $(419 \pm 1 \mu \mathrm{g} \mathrm{AA} e q / \mathrm{mL})$ and vitamin $\mathrm{C}$ content $(15,75$ $\pm 0,03 \mu \mathrm{g} / \mathrm{mL}$ ) and also a significant content of phenolic compounds (7,6 $\pm 2 \mathrm{mg}$ AGE/mL). Likewise, the results of the sensory panel showed that beverage 2 had a high overall acceptability and a greater acceptance of the taste, so it can be concluded that this drink is the one with the greatest antioxidant and commercial potential.

Keywords: Aloe vera (L.) Burm. F.; Aloin; Phenolic compounds; Anti-radical capacity; Sensory panel.

\section{INTRODUCCIÓN}

Las bebidas energizantes son no alcohólicas que, típicamente, contienen compuestos, como la cafeína, en concentraciones mayores a $150 \mathrm{mg} / \mathrm{L}$ y altas cantidades de azúcar, en combinación con otros ingredientes, que tienen propiedades estimulantes (Visram et al. 2016). En el mercado global son diversos los productos de este tipo que se pueden encontrar, teniendo una aceptación entre la población, por su capacidad para aliviar la fatiga, mantener la vigilia, mejorar el rendimiento físico y estimular las capacidades cognitivas, ante situaciones de estrés (Sánchez et al. 2015). Se comercializan, explícitamente, como una forma de aliviar la fatiga y mejorar la agudeza mental, en contraste con las bebidas deportivas o isotónicas, que tienen por objeto ayudar a los atletas a rehidratarse después del ejercicio.

Por otra parte, también es evidente que, entre la población, hay un creciente interés sobre productos más saludables o con propiedades nutracéuticas (López Molina et al. 2016). La panela es un edulcorante natural, obtenido del jugo de caña y con aporte de actividad antioxidante, debido a la presencia de compuestos polifenólicos, además, aportando micronutrientes, como calcio, magnesio, potasio, fósforo, sodio, hierro, manganeso, zinc y cobre, así como vitaminas A, B 1, B2, B5, B6, C, D y E (López-Palacios et al. 2019).

Las tendencias del mundo actual en bebidas de frutas exóticas tienen como objetivo demostrar una bioactividad significativa, con impacto positivo sobre varios trastornos crónicos (Stafussa et al. 2018). En este sentido, las investigaciones se han centrado en la combinación de frutas exóticas con otros ingredientes en bebidas, probablemente, debido a la asociación de los jugos de frutas, como una fuente natural de nutrientes y de fitoquímicos, como los compuestos fenólicos, para una buena salud (Carbonell-Capella et al. 2015). Asimismo, la producción de otros tipos de bebidas con adición de cristal o jugo de aloe vera (Aloe vera (L.) Burm.f.), ha sido un tema de interés para la comunidad científica, por sus propiedades nutricionales (Rodríguez Villacis et al. 2019) y por sus efectos en el tratamiento de enfermedades, como artritis, cáncer, diabetes y enfermedades del corazón (Nazir \& Ahsan, 2017). Se ha encontrado que cuando se rebanan las hojas para extraer el gel de aloe vera, se lesionan los haces vasculares, liberando una sustancia que se llama aloína, compuesta por antraquinonas que, en altas concentraciones, pueden irritar el tracto gastrointestinal, produciendo un efecto laxante (Cedillo \& Gastélum-Barrios, 2015). Esta sustancia puede ser reducida hasta niveles imperceptibles, con repetitivos lavados con agua caliente o mediante el uso de carbón activado (Martínez et al. 2017).

El objetivo de este trabajo fue desarrollar una bebida energizante con propiedades antioxidantes, a base de panela, jugo de maracuyá y cristales de aloe vera (Aloe vera (L.) Burm. F.).

\section{MATERIALES Y MÉTODOS}

Materiales. Para la preparación de todas las soluciones de las determinaciones químicas, se utilizó agua desionizada (Milli- ${ }^{\mathrm{TM}}$ ). Los reactivos utilizados fueron de grado analítico, marca Merck (USA) y los compuestos usados como estándares fueron de marca Sigma (USA).

Obtención del cristal de aloe vera. La obtención del cristal de aloe vera (AV), se realizó mediante el tratamiento de pencas de AV, cosechadas en una finca, ubicada en el municipio de Timbío, Cauca, de edades entre dos y cuatro años. A las pencas, se le cortaron los bordes, aproximadamente $1 \mathrm{~cm}$, se le retiró la epidermis y se extrajeron los trozos de la pulpa o gel de AV. Estos trozos, se sumergieron inmediatamente en agua y se le realizó un lavado con homogenización constante. Posteriormente, se realizaron tres lavados con agua caliente $55^{\circ} \mathrm{C}$ x 10 minutos, en una relación agua: aloe de 3:1. Estos tratamientos, se efectuaron con el objetivo de reducir el contenido de aloína y preservar las propiedades nutracéuticas del gel de AV (Sánchez-Machado et al. 2017). Luego del segundo lavado, se cortaron los trozos de AV en cubos de $0,5 \mathrm{~cm}^{3}$, aproximadamente, se homogenizaron, se empacaron en bolsas de polipropileno y se almacenaron a $-18^{\circ} \mathrm{C}$.

Obtención del jugo de maracuyá. Los frutos de maracuyá (Passiflora edulis f. flavicarpa O. Deg.), se obtuvieron de un mercado local, realizando la selección, según las condiciones de calidad, que debe tener el fruto (NTC -1267/79 del ICONTEC). Una vez se realizaron los protocolos de limpieza y de desinfección, se extrajo la pulpa y, posteriormente, se trituró en una licuadora casera (Oster, USA). El jugo obtenido, se filtró y se empacó en bolsas de polipropileno y se almacenó a $-18^{\circ} \mathrm{C}$.

Panela y otros ingredientes. Se utilizó panela marca el Triunfo, producida por una asociación agropecuaria de productores y comercializadores paneleros del municipio de Cajibío, Cauca, Registro Invima $N^{\circ}$ RSAZ1GI2501. El agua utilizada fue marca Cristal (Postobón, Colombia), cafeína anhidra y ácido ascórbico, de grado alimentario. 
Caracterización funcional de las materias primas. Para determinar la actividad antioxidante en el cristal de AV, se realizó una trituración manual hasta obtener una fracción líquida; para la panela, se preparó una solución acuosa $5 \% \mathrm{~m} / \mathrm{v}$; el jugo de maracuyá, junto con las otras fracciones líquidas, se filtró con vacío sobre papel filtro y se tomaron alícuotas, para su caracterización.

Determinación de compuestos fenólicos. Las fracciones líquidas de las materias primas, se utilizaron para la determinación de compuestos fenólicos. Se utilizó el método espectrofotométrico del Reactivo de Folin-Ciocalteu, siguiendo el procedimiento reportado en Nieto Calvache et al. (2016). Se realizó una curva de calibración, con una solución de ácido gálico $0,2 \% \mathrm{~m} / \mathrm{v}$ y se midió la absorbancia, a una longitud de onda de $765 \mathrm{~nm}$. Los resultados, se expresaron como equivalentes de ácido gálico.

Determinación de la capacidad antirradicalaria. La medición de la capacidad antirradicalaria de las materias primas, se realizó a través del método del radical libre 2,2-difenil-1-picrilhidrazil (DPPH). Este ensayo permite medir la capacidad de los compuestos presentes en una muestra, para actuar como captadores de radicales libres. Se utilizó una modificación de la técnica informada por Nieto-Calvache et al. (2018), registrándose el cambio de absorbancia en el tiempo. Se construyó una curva de calibración, con una solución de ácido ascórbico $200 \mu \mathrm{M}$. Brevemente, $0,1 \mathrm{~mL}$ de cada solución, se mezclaron con $3,9 \mathrm{~mL}$ de solución DPPH, $6 \times 10^{-5} \mathrm{M}$, preparada en etanol $(96 \mathrm{~mL} / 100 \mathrm{~mL})$ y se incubó durante 30 minutos; posteriormente, se midió la variación en la absorbancia $(K=515 \mathrm{~nm})$. Los resultados, se reportaron como actividad antirradicalaria equivalente a la del ácido ascórbico.

Proceso de producción de las bebidas. Se prepararon $500 \mathrm{~mL}$ de diferentes bebidas, con distintos porcentajes de AV, jugo de maracuyá y panela, de acuerdo con un diseño experimental de mezclas. Para la preparación, el agua, junto con el jugo de maracuyá y la panela, se homogenizaron con una licuadora de mano (Philips HR1623/00), durante 2 minutos. Posteriormente, se adicionaron los cristales de AV. A la mezcla obtenida, se le realizó un proceso de pasteurización lenta, a $70^{\circ} \mathrm{C}, 25 \mathrm{~min}$ (Petruzzi et al. 2017); las bebidas, se envasaron en recipientes plásticos y se almacenaron a $2^{\circ} \mathrm{C}$.

Caracterización química de las bebidas. Las bebidas desarrolladas, se analizaron en cuanto a su contenido de compuestos fenólicos y a su capacidad antirradicalaria a las 24 horas, utilizando los mismos protocolos, mencionados anteriormente, para las materias primas. Adicionalmente, se realizó una determinación de vitamina $C$, según el método cromatográfico oficial AOAC 2012.21. La determinación, se hizo en un equipo SHIMADZU LC 2010A HT, con una columna PHENOMENEX LUNA ${ }^{\circledR}$ C-18, de tamaño de partícula de $5 \mu \mathrm{m}$ y una fase móvil de acetonitrilo, con un flujo de $1 \mathrm{~mL} / \mathrm{min}$. Se realizó una curva de calibración, con soluciones estándar de ácido ascórbico.

Finalmente, se realizó una cuantificación del contenido de aloína, mediante método cromatográfico (HPLC), de acuerdo con Brown et al. (2014). Esta determinación fue realizada por el Laboratorio de Análisis Industrial y de alimentos, Angel Bioindustrial, como servicio externo.
Análisis sensorial. Luego del análisis del diseño experimental, se eligieron tres formulaciones, teniendo en cuenta las de mayor contenido de compuestos fenólicos y capacidad antirradicalaria. Con estas bebidas, se efectuó un análisis sensorial, para verificar la aceptación de 4 atributos sensoriales, mediante una escala hedónica de nueve puntos, donde cada número significa: 1 me disgusta muchísimo, 2 me disgusta mucho, 3 me disgusta moderadamente, 4 me disgusta un poco, 5 ni me gusta ni me disgusta, 6 me gusta un poco, 7 me gusta moderadamente, 8 me gusta mucho, 9 me gusta muchísimo. El análisis fue realizado por 171 panelistas (consumidores habituales de este tipo de bebidas), quienes evaluaron el sabor, textura del AV en la boca, color y aceptabilidad global, teniendo en cuenta las recomendaciones de Lawless (2013). Para este ensayo, se prepararon las bebidas adicionando $280 \mathrm{mg} / \mathrm{L}$ de cafeína grado alimentario y $50 \mathrm{mg} / \mathrm{Kg}$ ácido ascórbico grado alimentario, como antioxidante, cumpliendo con las cantidades máximas sugeridas por la Resolución 4150 DE 2009 - Ministerio de la Protección Social (2009), respectivamente, para este tipo de bebidas.

Diseño experimental y análisis estadístico. Los rangos de los porcentajes de panela, de maracuyá y de AV, se establecieron de acuerdo con ensayos sensoriales preliminares (datos no mostrados). Se utilizó un diseño de mezclas simplex con centroide, donde se consideraron los porcentajes de: panela, jugo de maracuyá y aloe vera, como factores experimentales y el contenido de compuestos fenólicos y capacidad antirradicalaria, como variables respuesta. En la tabla 2, se muestran los niveles de cada factor en sus valores reales y codificados. Se verificó el ajuste de los resultados a modelos polinómicos, para la creación de las superficies de respuesta, mediante un análisis de varianza $(p<0,05)$ y el estadístico $R^{2}$.

Modelo lineal

$$
E(y)=\sum_{i=1}^{q} \beta_{i} X_{i}+e_{u}
$$

Modelo cuadrático

$$
E(y)=\sum_{i=1}^{q} \beta_{i} X_{i}+\sum \sum_{i<j}^{q} \beta_{i j} X_{i} X_{j}+e_{u}
$$

Modelo cúbico especial

$$
E(y)=\sum_{i=1}^{q} \beta_{i} X_{i}+\sum \sum_{i<j=2}^{q} \beta_{i j} X_{i} X_{j}+\sum \sum_{i<j<k=2} \sum^{q} \beta_{i j k} X_{i} X_{j} X_{k}+e_{u}
$$

Donde, $E(y)$ es la respuesta y $e_{u}$ es el error en el $u$-ésimo ensayo; el parámetro $\beta_{i}$ representa el efecto del i-ésimo componente puro, $\beta_{i j}$ el efecto de la interacción entre los componentes $i$ y j y $\beta_{i j k}$ el efecto de la interacción triple entre los componentes $i$,jy k (Gutiérrez-Pulido \& de la Vara-Salazar, 2008).

Para el planteamiento y el análisis del diseño experimental, se utilizó el software Statgraphics Centurion XV (02/15/06 Versión, 2007 Statpoint Inc, Herndon, VA, Canada). Asimismo, para la 
comparación entre los valores de las propiedades fisicoquímicas y los resultados del análisis sensorial de las bebidas seleccionadas, se realizó un ANOVA de una vía, con un Test de Tukey post hoc, utilizando el utilitario del software GraphPad Prism versión 5.00 para Windows (San Diego, CA, USA).

\section{RESULTADOS Y DISCUSIÓN}

Análisis de materias primas. El análisis de las materias primas demostró que, tanto el AV como la pulpa de maracuyá y la panela, presentaban contenido de compuestos fenólicos, siendo la panela la que mostró los resultados más altos de las materias primas (Tabla 1). En estudios realizados por De Maria (2013), se han reportado valores para compuestos fenólicos entre 20 y 50mg AGE/g, en panela, de países como Japón, India, Francia y Venezuela, lo cual, está en el orden de los valores encontrados en la panela utilizada en este trabajo.
El AV, se considera un ingrediente importante para la elaboración de alimentos funcionales, debido a su aporte de polisacáridos de la fibra dietaria y compuestos con actividad antioxidante, como los fenólicos (Vega et al. 2005). En AV, se ha reportado capacidad antirradicalaria, efectiva para la estabilización de los radicales DPPH y el 2,2-azinobis-3etil benzotioazolín-6-sulfónico (ABTS) (Radha \& Laxmipriya, 2015). Por otra parte, en los trabajos de de Santana et al. (2017), se encontraron compuestos fenólicos en semillas de maracuyá, en concentraciones de 3,11 \pm 0,07g AGE/100g. Otros autores, como Rotta et al. (2019), determinaron diferentes tipos de compuestos fenólicos en la pulpa de maracuyá, hallando que los compuestos fenólicos, como el ácido vanílico y quercetina, eran los más abundantes y que, además, esta pulpa tenía capacidad antirradicalaria, mediante el método del DPPH, en el orden de $800 \mu g$, equivalentes de Trolox $/ 100 \mathrm{~g}$, otro antioxidante muy utilizado en la literatura, como patrón antioxidante.

Tabla 1. Contenido de compuestos fenólicos y capacidad antirradicalaria en las materias primas principales.

\begin{tabular}{|c|c|c|c|}
\hline & Panela (mg AGE/g) & Jugo maracuyá (mg AGE/mL) & Aloe vera $(\mathrm{mg} \mathrm{AGE} / \mathrm{mL})^{1}$ \\
\hline Compuestos Fenólicos & $59,4 \pm 0,2$ & $7,7 \pm 0,2$ & $0,57 \pm 0,09$ \\
\hline & Panela $(\mu \mathrm{g} \text { eq } \mathrm{AA} / \mathrm{g})^{2}$ & Jugo maracuyá ( $\mu$ g eq $\mathrm{AA} / \mathrm{mL})^{2}$ & Aloe vera $(\mu \mathrm{g} \text { eq } \mathrm{AA} / \mathrm{mL})^{2}$ \\
\hline Capacidad Antirradicalaria & $25,0 \pm 0,1$ & $657 \pm 5$ & $27,9 \pm 0,1$ \\
\hline
\end{tabular}

${ }^{1}$ AGE: significa Equivalentes de ácido gálico; ${ }^{2}$ Significa capacidad antirradicalaria equivalente a microgramos de ácido ascórbico.

Análisis de las bebidas. El diseño experimental de mezclas es una herramienta estadística que ha sido utilizada en la formulación de mezclas alimenticias, para optimizar el porcentaje adecuado de ingredientes, respecto a una o varias variables de respuesta
(Salamanca Grosso et al. 2015). Los resultados de las propiedades antioxidantes de las bebidas obtenidas, se resumen en la tabla 2. Se encontró un contenido de compuestos fenólicos, que osciló entre 5,5 y 9,58mg AGE/mL. Asimismo, los resultados de la capacidad antirradicalaria oscilaron entre 371 y $408 \mu \mathrm{g}$ eq AA/mL.

Tabla 2. Diseño experimental de mezclas, con sus valores codificados y reales y con los resultados de los compuestos fenólicos y la capacidad antirradicalaria.

\begin{tabular}{|c|c|c|c|c|c|}
\hline Sistema & Panela & $\begin{array}{l}\text { Pulpa } \\
\text { maracuyá }\end{array}$ & Aloe vera & $\begin{array}{c}\text { Compuestos } \\
\text { Fenólicos } \\
(\mathrm{mg} \mathrm{AGE} / \mathrm{mL})^{1}\end{array}$ & $\begin{array}{c}\text { Capacidad antirradicalaria } \\
(\mu \mathrm{g} \text { eq } \mathrm{AA} / \mathrm{mL})^{2}\end{array}$ \\
\hline 1 & $1(9)$ & $0(5)$ & $0(5)$ & $9,58 \pm 0,05$ & $371,96 \pm 0,03$ \\
\hline 2 & $0(6)$ & $1(7)$ & $0(5)$ & $7,47 \pm 0,09$ & $408 \pm 1$ \\
\hline 3 & $0(6)$ & $0(5)$ & $1(8)$ & $5,89 \pm 0,19$ & $400 \pm 4$ \\
\hline 4 & $0,5(7,5)$ & $0,5(6)$ & $0(5)$ & $6,87 \pm 0,04$ & $395,3 \pm 0,5$ \\
\hline 5 & $0,5(7,5)$ & $0(5)$ & $0,5(6,5)$ & $7,33 \pm 0,02$ & $380 \pm 2$ \\
\hline 6 & $0(6)$ & $0,5(6)$ & $0,5(6,5)$ & $5,5 \pm 0,2$ & $403,5 \pm 0,3$ \\
\hline 7 & $0,333(7)$ & $0,333(5,66)$ & $0,333(6)$ & $6,5 \pm 0,5$ & $384 \pm 1$ \\
\hline 8 & $0,667(8)$ & $0,167(5,33)$ & $0,167(5,5)$ & $7,0 \pm 0,5$ & $383,1 \pm 0,2$ \\
\hline 9 & $0,167(6,5)$ & $0,667(6,33)$ & $0,167(5,5)$ & $5,8 \pm 0,3$ & $402,9 \pm 0,6$ \\
\hline 10 & $0,167(6,5)$ & $0,167(5,33)$ & $0,667(7)$ & $6,070 \pm 0,008$ & $396 \pm 2$ \\
\hline
\end{tabular}

${ }^{1} \mathrm{AGE}$ significa ácido gálico equivalente; ${ }^{2} \mathrm{AA}$ significa ácido ascórbico equivalente. 
Por otra parte, se realizó la optimización de cada una de las propiedades, con el objetivo de maximizar sus respuestas. El análisis de los resultados de la composición de compuestos fenólicos de las bebidas demostró que los datos, para compuestos fenólicos, ajustaron a un modelo cuadrático, $\mathrm{CF}=9,47626 * \mathrm{P}+7,38034 * \mathrm{M}$ + 5,97415*AV - 6,99983*P*M - 1,66064*P*AV - 4,49674*M*AV, donde, CF: Compuestos fenólicos, P: panela, M: maracuyá y AV: aloe vera. El valor p obtenido en el ANOVA fue de 0,0067, lo cual, indica que existe una relación estadísticamente significativa entre el contenido de compuestos fenólicos y los componentes de la mezcla, con un nivel de confianza del $95 \%$. El análisis del estadístico $\mathrm{R}^{2}$ indicó que el modelo explica un $96 \%$ de la variabilidad de los compuestos fenólicos, según este modelo.
En la figura 1, se muestra la superficie de respuesta para el contenido de compuestos fenólicos de las bebidas producidas. Se puede observar que, cuando el porcentaje de panela o maracuyá es alto, se detecta un mayor contenido de fenólicos, mientras que con los valores de la mezcla pura de aloe vera, se detectan menores contenidos. Este resultado es acorde con el análisis realizado para las materias primas (Tabla 1), donde se había encontrado que la panela hacía el mayor aporte de compuestos fenólicos, seguido de pulpa de maracuyá.

En cuanto a la capacidad antirradicalaria, también los datos ajustaron a un modelo cuadrático: $\mathrm{CA}=372,72 * \mathrm{P}+409,131 * \mathrm{M}+400,874 * \mathrm{AV}$ $+12,8466 * \mathrm{P} * \mathrm{M}-31,6299 *$ Panela*AV - 9,99267*M*AV, donde CA,

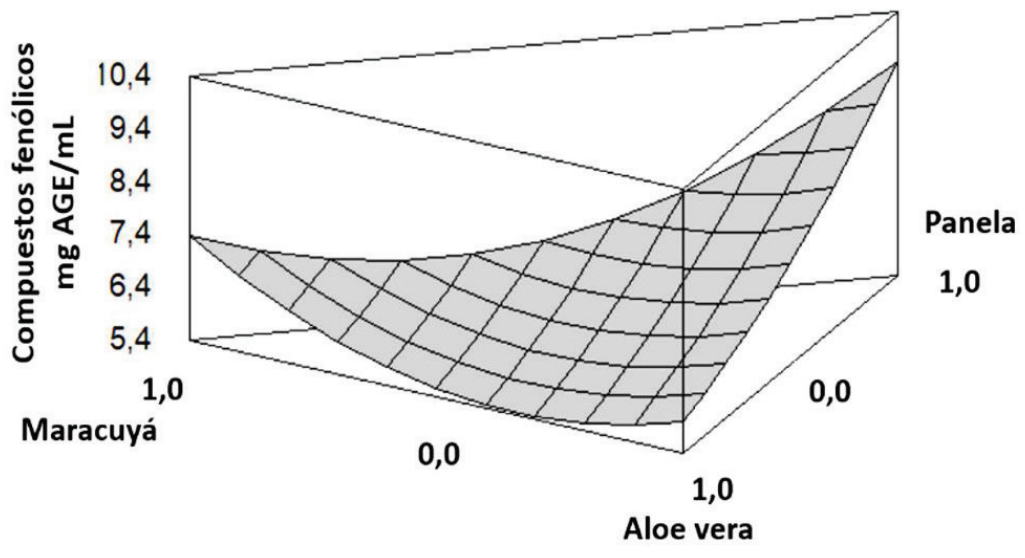

Figura 1. Superficie de respuesta, representando el efecto de las materias primas sobre los compuestos fenólicos de las bebidas.

es la capacidad antirradicalaria. El valor p, para este modelo, fue de 0,0113, denotando una relación estadísticamente significativa entre la capacidad antirradicalaria y los componentes de la mezcla, con un nivel de confianza del 95,0\%. Además, el valor del $\mathrm{R}^{2}$ fue del $94,8 \%$, explicando un alto porcentaje de la variabilidad de los datos de la capacidad antirradicalaria. La figura 2 muestra la superficie de respuesta de la capacidad antirradicalaria; se puede observar, que la mayor actividad antirradicalaria es aportada por la pulpa de maracuyá y el AV, mientras que la panela es aquella que hace el menor aporte de la capacidad antirradicalaria, por lo cual, se puede inferir que, además de los compuestos fenólicos, las materias primas contienen otro tipo de compuestos, que contribuyen con la capacidad antirradicalaria.

Mediante este análisis estadístico, se obtuvo que los porcentajes de las materias primas, óptimos para maximizar las dos variables respuesta era diferente (Tabla 3). Se obtuvo un sistema que permite obtener una bebida 1 optimizada, para contener un máximo de compuestos fenólicos y otro, para obtener una bebida 2 optimizada, para tener la mayor capacidad antirradicalaria (Tabla 3). Adicionalmente, y para fines comparativos y estudios de profundización, se planteó una bebida adicional (bebida 3), utilizando los porcentajes estimados de panela y de maracuyá promedio, de las bebidas 1 y 2 y así obtener un sistema adicional, con propiedades antioxidantes intermedias.

Se produjeron los sistemas de bebidas óptimos e intermedio (Tabla 3), análogamente a lo realizado durante el diseño experimental, con los porcentajes de panela, jugo de maracuyá y aloe vera, estimados estadísticamente y se analizaron, una vez más, en cuanto al contenido de compuestos fenólicos y capacidad antirradicalaria; adicionalmente, se cuantificó el contenido de vitamina C. Los resultados de este análisis mostraron que la bebida que presentaba mayor capacidad antirradicalaria $(\mathrm{p}<0,05)$ era la bebida 2 , mostrando valores de $419 \pm 1 \mu \mathrm{g}$ eq AA/mL, seguido de la bebida 1 (399 \pm $4 \mu \mathrm{g}$ eq $\mathrm{AA} / \mathrm{mL})$ y la bebida $3(372 \pm 4 \mathrm{eq} \mathrm{AA} / \mathrm{mL})$. En cuanto al contenido de compuestos fenólicos, se encontró que la bebida 1 era la que contenía la mayor cantidad $(\mathrm{p}<0,05)$, mostrando valores de $8,5 \pm 0,3 \mathrm{mg} \mathrm{AGE} / \mathrm{mL}$, seguido de la bebida 2 (7,6 \pm 0,2mg AGE/ $\mathrm{mL})$ y de la bebida $3(7,3 \pm 0,1 \mathrm{mg} \mathrm{AGE} / \mathrm{mL})$. Adicionalmente, los resultados del análisis de vitamina $\mathrm{C}$ reportaron que el mayor contenido de vitamina $C(p<0,05)$ lo presentaba la bebida $2(15,75$ $\pm 0,03 \mu \mathrm{g} / \mathrm{mL})$, seguido por la bebida $3(15,25 \pm 0,03 \mu \mathrm{g} / \mathrm{mL})$ y por la bebida $1(14,80 \pm 0,07 \mu \mathrm{g} / \mathrm{mL})$, por lo cual, la mayor 


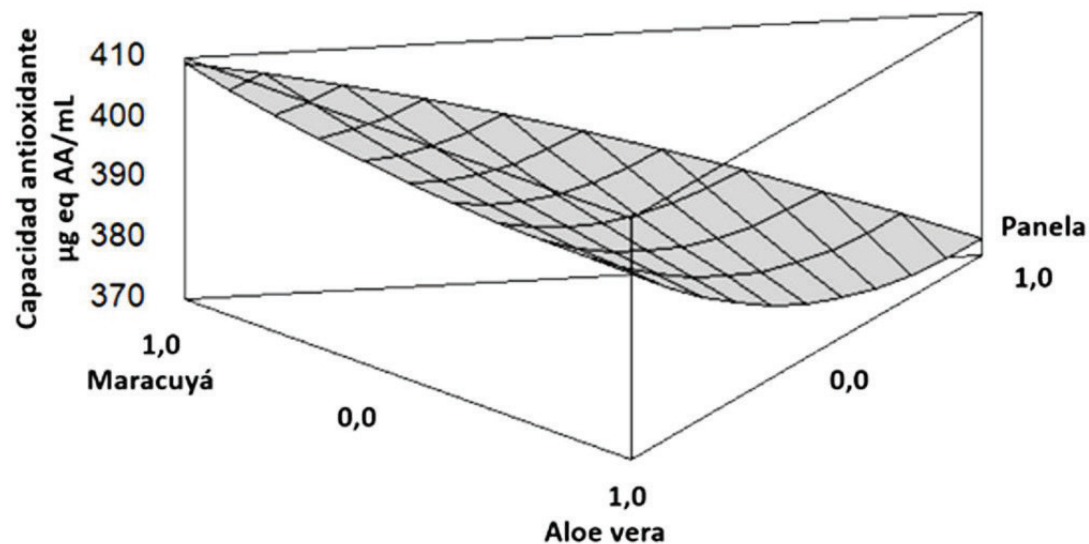

Figura 2. Superficie de respuesta, representando el efecto de los ingredientes sobre la capacidad antirradicalaria.

Tabla 3. Materias primas estimadas para la producción de las tres bebidas finales.

\begin{tabular}{|l|c|c|c|}
\hline & $\begin{array}{c}\text { Óptimo para fenólicos } \\
\text { (bebida 1) }\end{array}$ & $\begin{array}{c}\text { Óptimo para capacidad antirradicalaria } \\
\text { (bebida 2) }\end{array}$ & $\begin{array}{c}\text { Intermedio } \\
\text { (bebida 3) }\end{array}$ \\
\hline Panela (\%) & 9 & 6,6 & 7,8 \\
\hline Maracuyá (\%) & 5 & 7 & 6 \\
\hline Aloe vera (\%) & 5 & 5 & 5 \\
\hline & Compuestos fenólicos & $\begin{array}{c}\text { Capacidad } \\
\text { antirradicalaria }\end{array}$ & \\
\hline Valor estimado estadísticamente & $9,4 \mathrm{mg}$ AGE/mL & $401 \mu \mathrm{g}$ eq AA/mL & -- \\
\hline
\end{tabular}

actividad antirradicalaria de la bebida 2, puede estar asociada al mayor contenido de vitamina C. En los trabajos realizados por Sir Elkhatim et al. (2018), se ha demostrado una correlación positiva, tanto de la vitamina $\mathrm{C}$ como de los compuestos fenólicos, con la capacidad antirradicalaria. En diferentes trabajos científicos, generalmente, se reportan que los compuestos fenólicos son los mayores aportadores de actividad antioxidante (Manganaris et al. 2014); sin embargo, en otros tejidos vegetales, también se ha reportado que la vitamina $C$ tiene mayor poder antioxidante que los compuestos fenólicos (Sir Elkhatim et al. 2018). Estas diferencias en el poder antioxidante de los compuestos bioactivos están asociadas con el tipo de tejido vegetal, el estado de maduración, la variedad y las condiciones agronómicas del cultivo.

Finalmente, se determinó que el contenido de aloína en las 3 bebidas energizantes era menor a $0,1 \mathrm{mg} / \mathrm{Kg}$, cumpliendo con lo establecido en el Codex Alimentarius y la FAO (2008), Requisitos generales para aromatizantes naturales CAC/GL 29-1987, donde se establece que el aloe puede hacer parte de la composición de un alimento, siempre y cuando no se sobrepase los límites máximos permitidos de aloína: $0,1 \mathrm{mg} / \mathrm{kg}$, en el producto final, listo para el consumo en alimentos y bebidas. Estos resultados, también validan que los tratamientos hidrotérmicos realizados al AV permiten una reducción del contenido de aloína hasta los valores recomendados.
Análisis sensorial. El análisis sensorial permite verificar el grado de aceptación de los productos por panelistas, consumidores habituales de este tipo de bebidas. Las bebidas fueron preparadas con cafeína, ya que este es el componente que las clasifica como bebidas energizantes. La figura 3 resume los resultados del análisis sensorial. Se encontró que, estadísticamente, las tres bebidas fueron aceptadas por los panelistas, con valores por encima de 6, en la escala hedónica. En cuanto a la textura del aloe en la boca, se encontró que las tres bebidas fueron calificadas con valores significativamente iguales $(\mathrm{p}<0,05)$. En cuanto al sabor, se observó que las bebidas 1 y 2 tuvieron una mejor aceptación $(\mathrm{p}<0,05)$, que la bebida 3 . El color de las tres bebidas tuvo buena aceptación, sin denotar diferencias significativas $(p<0,05)$. Finalmente, en cuanto a la aceptabilidad global, las bebidas 1 y 2 tuvieron una mejor calificación $(\mathrm{p}<0,05)$, que la bebida 3 .

De acuerdo con los resultados, se puede concluir que, las bebidas desarrolladas, mostraron un importante contenido de compuestos bioactivos, aportados por las materias primas utilizadas, las cuales, otorgaron un valor funcional, como la capacidad antirradicalaria, aportada, principalmente, por la presencia de compuestos fenólicos y vitamina C. El análisis del panel sensorial, demostró que todas las bebidas fueron aceptadas por los consumidores; sin embargo, la 


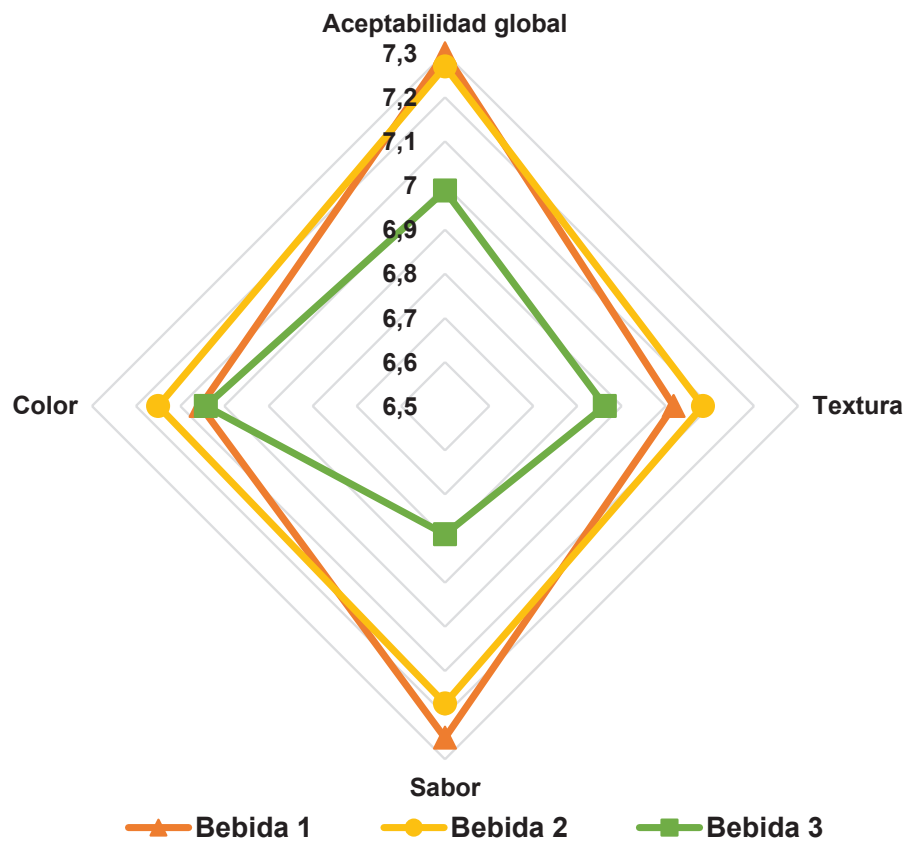

Figura 3. Análisis de la aceptación sensorial de las tres bebidas producidas.

bebida 2 fue la que presentó mayor potencial funcional, por su mayor capacidad antirradicalaria, su mayor contenido de vitamina $\mathrm{C}$ y un intermedio contenido de compuestos fenólicos. Además, esta bebida fue una de las que presentó mejor valoración en cuanto al sabor y la aceptabilidad global, presentando así un mejor potencial comercial.

Agradecimientos: Al Servicio Nacional de aprendizaje, SENA, por su soporte técnico y financiero, mediante el proyecto SGPS 3563. También, se agradece a la Universidad del Cauca, por el apoyo técnico, a través del grupo de investigación ASUBAGROIN. Conflicto de intereses: El manuscrito fue preparado y revisado con la participación de todos los autores, quienes declaramos que no existe ningún conflicto de intereses que ponga en riesgo la validez de los resultados presentados.

\section{REFERENCIAS}

1. BROWN, P.N.; YU, R.; KUAN, C.H.; FINLEY, J.; MUDGE, E.M.; DENTALI, S. 2014. Determination of Aloin A and Aloin B in Aloe vera raw materials and finished products by high-performance liquid chromatography: single-laboratory validation. J. AOAC Int. (Reino Unido). 97(5):1323-1328. https://doi.org/10.5740/jaoacint.13-028

2. CARBONELL-CAPELLA, J.M.; BUNIOWSKA, M.; ESTEVE, M.J.; FRÍGOLA, A. 2015. Effect of Stevia rebaudiana addition on bioaccessibility of bioactive compounds and antioxidant activity of beverages based on exotic fruits mixed with oat following simulated human digestion. Food Chem. (Holanda). 184:122-130.

https://doi.org/10.1016/j.foodchem.2015.03.095

3. CEDILLO, C.; GASTÉLUM-BARRIOS, A. 2015. Desarrollo tecnológico de remoción de aloína en sistema continuo de adsorción en columna. In Proceedings 3er. Congreso Internacional de Ingeniería y Tecnología, Universidad del SABES. San Miguel de Allende, Gto., México: Universidad del SABES. Disponible desde Internet en: https://www.researchgate.net/profile/Abraham_ Gastelum_Barrios/publication/314500750_ Desarrollo_tecnologico_de_remocion_de_ aloina_en_sistema_continuo_de_adsorcion_en_ columna_Diseno_y_perspectivas_de_monitoreo/ links /58c2c82d92851c0ccbf114e8/Desarrollo-tecno (con acceso 24/04/20).

4. CODEX/FAO. 2008. PROGRAMA CONJUNTO FAO/ OMS SOBRE NORMAS ALIMENTARIAS COMITÉ DEL CODEX SOBRE ADITIVOS ALIMENTARIOS $40^{a}$ reunión. Beijing (China). Disponible desde internet en: http:/ / www.fao.org/tempref/codex/Meetings/CCFA/ ccfa40/fa40_08s.pdf (con acceso 24/04/20). 
5. DE MARIA, G. 2013. Panela: The natural nutritional sweetener. Agro Food Ind Hi-Tec. (Italia). 24(6):44-48.

6. DE SANTANA, F.C.; DE OLIVEIRA TORRES, L.R.; SHINAGAWA, F.B.; DE OLIVEIRA E SILVA, A.M.; YOSHIME, L.T.; DE MELO, I.L.P.; MARCELLINI, P.S.; MANCINI-FILHO, J. 2017. Optimization of the antioxidant polyphenolic compounds extraction of yellow passion fruit seeds (Passiflora edulis Sims) by response surface methodology. J Food Sci and Tec. (India). 54(11):35523561.

https://doi.org/10.1007/s13197-017-2813-3

7. GUTIÉRREZ PULIDO, H.; DE LA VARA SALAZAR, R. 2008. Análisis y diseño de experimentos. McGraw-Hill Interamericana. (México DF). 488p.

8. LAWLESS, H. 2013. Quantitative Sensory Analysis. Psychophysics, Models and Intelligent Design. Ed. WileyBlackwell (Reino Unido).p.167-170.

9. LÓPEZ MOLINA, M.G.; CRUZ VÁSQUEZ, M.; BUITRÓN MORALES, P.; DE LAROCHA SOSA, A. 2016. Modelo de negocio de alimentos procesados saludables: Estudio de caso con análisis de la propuesta de valor. Strateg. Technol. \& Soc. (México). 3(2):43-68.

10. LÓPEZ-PALACIOS, K.; GONZALES-CORTÉS, N.; MALDONADO-ENRIQUEZ, A.; JIMENEZ，L.; JIMENEZ-VERA, R. 2019. Jugo de betabel (Beta vulgaris L.) y panela fermentados con Saccharomyces bayanus. In Crescendo. (Peru). 9(3):367-378.

11. MANGANARIS, G.A.; GOULAS, V.; VICENTE, A.R.; TERRY, L.A. 2014. Berry antioxidants: Small fruits providing large benefits. J. Sci. Food Agric. (Estados Unidos). 94(5):825-833.

https://doi.org/10.1002/jsfa.6432

12. MARTÍNEZ, W.J.; PATERNINA-ARBOLEDA, C.D.; PARODDY, A.; VÉLEZ RAMÍREZ, M.; TAPIA, L.P.; ESPITIA, R. 2017. Optimización del proceso de eliminación de aloína del gel fileteado mecánico de Aloe vera concentrado 10X. Cumbres. (Ecuador). 3(2):9-16.

13. MINISTERIO DE LA PROTECCIÓN SOCIAL. 2009. RESOLUCIÓN 4150 DE 2009. Por la cual se establece el reglamento técnico sobre los requisitos que deben cumplir las bebidas energizantes para consumo humano. Disponible desde Internet en:

https: / / www.minsalud.gov.co/sites/rid/Lists / BibliotecaDigital/RIDE/DE/DIJ/Resolucion-4150de-2009.pdf (con acceso 09/07/2020)
14. NAZIR, A.; AHSAN, H. 2017. Health benefits of aloe vera : A wonder plant. Int. J. Chem. Stud. (India). 5(6):967-969.

15. NIETO CALVACHE, J.E.; CUETO, M.; FARRONI, A.; DE ESCALADA PLA, M.F.; GERSCHENSON, L.N. 2016. Antioxidant characterization of new dietary fiber concentrates from papaya pulp and peel (Carica papaya L.). J. Funct. Foods. (Holanda). 27:319-328. http://dx.doi.org/10.1016/j.jff.2016.09.012

16. NIETO-CALVACHE, J.E.; DE ESCALADA PLA, M.; GERSCHENSON, L.N. 2018. Dietary fibre concentrates produced from papaya by-products for agroindustrial waste valorisation. Int. J. Food Sci. Technol. (Reino Unido). 1:1-7. https://doi.org/10.1111/ijfs.13962

17. PETRUZZI, L.; CAMPANIELLO, D.; SPERANZA, B.; CORBO, M.R.; SINIGAGLIA, M.; BEVILACQUA, A. 2017. Thermal treatments for fruit and vegetable juices and beverages: A literature overview. Compr. Rev. Food Sci. Food Saf. (Estados Unidos). 16(4):668-691. https://doi.org/10.1111/1541-4337.12270

18. RADHA, M.H.; LAXMIPRIYA, N.P. 2015. Evaluation of biological properties and clinical effectiveness of Aloe vera: A systematic review. J. Tradit. Complement. Med. (Holanda) 5(1):21-26. https://doi.org/10.1016/j.jtcme.2014.10.006

19. RODRÍGUEZ VILLACIS, D.; HERNÁNDEZ MONZON, A.; RODRÍGUEZ SANCHEZ, J.L. 2019. Caracterización de una bebida fermentada de lactosuero con la adición de jugo de sábila (Aloe vera L.) y pulpa de mora (Rubus glaucus Bent). Cienc. y Tecnol. Aliment. (Cuba). 29(1):7-14.

20. ROTTA, E.M.; RODRIGUES, C.A.; JARDIM, I.C.S.F.; MALDANER， L.; VISENTAINER, J.V. 2019. Determination of phenolic compounds and antioxidant activity in passion fruit pulp (Passiflora spp.) using a modified QuEChERS method and UHPLC-MS/MS. LWT. (Estados Unidos). 100:397-403.

https://doi.org/10.1016/J.LWT.2018.10.052

21. SALAMANCA GROSSO, G.; REYES MÉNDEZ, L.M.; OSORIO TANGARIFE, M.P.; RODRÍGUEZ ARIAS, N. 2015. Diseño experimental de mezclas como herramienta para la optimización de cremolácteos de mango. Rev. Col. Investig. Agroindustriales. 2:16-24. https://doi.org/10.23850/24220582.166

22. SÁNCHEZ, J.C.; ROMERO, C.R.; ARROYAVE, C.D.; GARCÍA, A.M.; GIRALDO, F.D.; SÁNCHEZ, L.V. 2015. Bebidas energizantes: efectos benéficos y perjudiciales para la salud. Perspect. Nutr. Humana. (Colombia). 17(1):79-91. https://doi.org/10.17533/udea.penh.v17n1a07 
23. SÁNCHEZ-MACHADO, D.I.; LÓPEZ-CERVANTES, J.; SENDÓN, R.; SANCHES-SILVA, A. 2017. Aloe vera: Ancient knowledge with new frontiers. Trends Food Sci Technol. (Holanda) 61:94-102.

https://doi.org/10.1016/j.tifs.2016.12.005

24. SIR ELKHATIM, K.A.; ELAGIB, R.A.A.; HASSAN, A.B. 2018. Content of phenolic compounds and vitamin $C$ and antioxidant activity in wasted parts of Sudanese citrus fruits. Food Sci. Nutr. (Reino Unido). 6(5):1214-1219. https://doi.org/10.1002/fsn3.660

25. STAFUSSA, A.P.; MACIEL, G.M.; RAMPAZZO, V.; BONA, E.; MAKARA, C.N.; DEMCZUK JUNIOR, B.; HAMINIUK, C.W.I. 2018. Bioactive compounds of 44 traditional and exotic brazilian fruit pulps: Phenolic compounds and antioxidant activity. Int. J. Food Prop. (Estados Unidos). 21(1):106-118.

https://doi.org/10.1080/10942912.2017.1409761
26. VEGA G, A.; AMPUERO C, N.; DÍAZ N, L.; LEMUS M, R. 2005. Aloe vera (Aloe barbadensis Miller) as a component of functional foods. Rev. Chil. Nutr (Chile) 32(3):208-214. http://dx.doi.org/10.4067/S0717-75182005000300005

27. VISRAM, S.; CHEETHAM, M.; RIBY, D.M.; CROSSLEY, S.J.; LAKE, A.A. 2016. Consumption of energy drinks by children and young people: A rapid review examining evidence of physical effects and consumer attitudes. BMJ Open. (Reino Unido). 6(10):e010380. https://doi.org/10.1136/bmjopen-2015-010380 